\title{
Metformin and lactic acidosis during shock: just the tip of the iceberg?
}

Rene A. Posma ${ }^{1 *}$, Anthony R. Absalom², Daan J. Touw ${ }^{3}$, Iwan C. C. van der Horst ${ }^{1}$ and Maarten W. N. Nijsten ${ }^{1}$

See related research by Doenyas-Barak et al. http://ccforum.biomedcentral.com/articles/10.1186/s13054-015-1180-6

\section{Letter}

Doenyas-Barak et al. concluded recently in Critical Care that with similar high lactate levels $(>10 \mathrm{mmol} / \mathrm{L})$ the prognosis of metformin users compared with non-users was favorable during admission to the emergency department with septic shock (in-hospital mortality, $57 \%$ versus $88 \%, P<0.001$ ) [1]. This observation and similar findings reported previously are suggestive of a role for metformin as a lactate generation amplifier [2, 3]. However, in this study, causality cannot be proven, since without metformin concentration measurements a doseresponse analysis is not possible.

Despite the dramatic clinical presentation of metformin-associated lactic acidosis (MALA), this study also underlines the fact that its prognosis is better than that of similarly severe lactic acidosis of other origins $[1,4]$. With an incidence of approximately 5 cases per 100,000 patient-years, MALA is considered an exceedingly rare complication of chronic metformin therapy [5]. Metformin is excreted unchanged by the kidneys, predisposing patients with renal insufficiency to the development of MALA. Several authors suggest that the inclusion of renal impairment as a contraindication for metformin use should be reconsidered, and this may increase the number of patients at risk for developing MALA [5].
The findings reported in this study shed new light on our current understanding of the impact of metformin on lactate production. Given the large difference in mortality between the two groups, it can be concluded that the prognostic value of lactate is largely altered by metformin use in patients presenting with shock. Evidently, a relatively small sample size of patients (44 metformin users and 118 non-users) is sufficient to observe differences of such a magnitude in survival. Although the effect may not be as pronounced as in the present study, we believe that metformin may also elevate lactate levels in patients presenting with less severe lactic acidosis $[1,2]$. Therefore, a study comparing the prognosis of critically ill metformin users with non-users in a large matched cohort based on increasing lactate levels seems useful.

Because the effect of metformin on lactate levels in such a population may not be immediately obvious, we suspect that hyperlactatemia partly induced by metformin is often not considered by physicians caring for patients presenting with critical conditions. This study might therefore have identified only the tip of the iceberg regarding the effect of metformin on lactate levels. For clinicians to be able to identify and treat MALA in a more timely manner, lactate levels should always be measured in the emergency department or even in the prehospital setting.

\section{Authors' response}

Keren Doenyas-Barak and Shai Efrati

The main finding of our study was that patients under metformin treatment with severe lactic acidosis and septic shock have better prognosis than patients without metformin treatment [1]. The difference can

\footnotetext{
* Correspondence: r.a.posma@umcg.nl

${ }^{1}$ Department of Critical Care, University Medical Center Groningen, University of Groningen, Groningen, The Netherlands

Full list of author information is available at the end of the article
}

be attributed to metformin accumulation, representing MALA, and not the severity of the sepsis. In such cases, the blood level of metformin would be expected to be high. However, the difference between the groups may also represent a metformin-related protective effect, in which case the blood level of metformin would not necessarily be high. Thus, lack of knowledge regarding 
circulating metformin levels is indeed a limitation of the present study. Nonetheless, it is clear that severe lactic acidosis under metformin treatment should be regarded as a different entity. Thus, we agree that subclinical MALA may be uncovered (or exposed) in some patients if larger populations are evaluated and if blood lactate is measured in all metformin users.

Posma et al. express concern that the low incidence of MALA may change if renal failure as a contraindication is reconsidered [6]. However, in the present cohort, acute rather than chronic renal failure was associated with MALA [1]. Metformin accumulates in patients with renal failure, and its plasma level was found to be in good correlation with renal function [7]. Thus, low-dose metformin treatment in patients with stable chronic renal failure may be safe. However, acute renal failure may pose the risk of acute metformin accumulation and lactic acidosis [8]. Thus, the risk for acute renal failure should always be considered before prescribing metformin.

\section{Abbreviations}

CKD: Chronic kidney disease; MALA: Metformin-associated lactic acidosis.

Authors' contributions

RAP wrote the manuscript. All authors participated in drafting the manuscript and read and approved the final version.

\section{Competing interests}

The authors declare that they have no competing interests.

\section{Author details}

${ }^{1}$ Department of Critical Care, University Medical Center Groningen, University of Groningen, Groningen, The Netherlands. ²Department of Anesthesiology, University Medical Center Groningen, University of Groningen, Groningen, The Netherlands. 'Department of Clinical Pharmacy and Pharmacology, University Medical Center Groningen, University of Groningen, Groningen, The Netherlands.

Published online: 01 June 2016

\section{References}

1. Doenyas-Barak K, Beberashvili I, Marcus R, Efrati S. Lactic acidosis and severe septic shock in metformin users: a cohort study. Crit Care. 2016;20:10.

2. Green JP, Berger T, Garg N, et al. Impact of metformin use on the prognostic value of lactate in sepsis. Am J Emerg Med. 2012;30:1667-73.

3. Seidowsky A, Nseir S, Houdret N, Fourrier F. Metformin-associated lactic acidosis: a prognostic and therapeutic study. Crit Care Med 2009;37:2191-6.

4. Friesecke $S$, Abel P, Roser M, Felix SB, Runge S. Outcome of severe lactic acidosis associated with metformin accumulation. Crit Care. 2010;14:R226.

5. Lalau JD, Arnouts P, Sharif A, De Broe ME. Metformin and other antidiabetic agents in renal failure patients. Kidney Int. 2015;87:308-22.

6. O'Brien RC, Adam WR. A justification for less restrictive guidelines on the use of metformin in stable chronic renal disease. Diabet Med. 2014;31:1032-8

7. Tucker GT, Cadey C, Phillips PJ, Connor H, Ward JD, Woods HF. Metformin kinetics in healthy subjects and in patients with diabetes mellitus. Br J Clin Pharmacol. 1981;12:235-46.

8. Cucchiari D, Podesta MA, Merizzoli E, Calvetta A, Morenghi E, Angelini C, et al. Dose-related effect of metformin on acid base balance and renal function in patients with diabetes who develop acute renal failure: a cross-sectional study. Acta Diabetol. 2016 Jan 28. [Epub ahead of print]. 